\title{
Trapping cold molecular hydrogen
}

\section{Journal Article}

\section{Author(s):}

Seiler, Christian; Hogan, Stephen D.; Merkt, Frédéric

Publication date:

2011-11-14

\section{Permanent link:}

https://doi.org/10.3929/ethz-a-010781593

Rights / license:

In Copyright - Non-Commercial Use Permitted

Originally published in:

Physical Chemistry Chemical Physics 13(42), https://doi.org/10.1039/C1CP21276A

Funding acknowledgement:

132688 - Zeeman and Stark deceleration of atoms and molecules (SNF) 
This article may be downloaded for personal use only. Any other use requires prior permission of the author and The Royal Society of Chemistry.

The following article appeared in Phys. Chem. Chem. Phys. 13, 19000-19012 (2011) and may be found at http://dx.doi.org/10.1039/C1CP21276A. 


\title{
Trapping Cold Molecular Hydrogen
}

\author{
Ch. Seiler, S. D. Hogan and F. Merkt ${ }^{a}$
}

\author{
Received Xth $X X X X X X X X X X 20 X X$, Accepted $X$ th $X X X X X X X X X 20 X X$ \\ First published on the web $X$ th $X X X X X X X X X X 200 X$ \\ DOI: 10.1039/b000000x
}

Translationally cold $\mathrm{H}_{2}$ molecules excited to non-penetrating $\left|M_{J}\right|=3$ Rydberg states of principal quantum number in the range $21-37$ have been decelerated and trapped using time-dependent inhomogeneous electric fields. The $\left|M_{J}\right|=3$ Rydberg states were prepared from the $\mathrm{X}^{1} \Sigma_{\mathrm{g}}^{+}(v=0, J=0)$ ground state using a resonant three-photon excitation sequence via the $\mathrm{B}^{1} \Sigma_{\mathrm{u}}^{+}(v=$ $3, J=1)$ and $\mathrm{I}^{1} \Pi_{\mathrm{g}}(v=0, J=2)$ intermediate states and circularly polarized laser radiation. The circular polarization of the VUV radiation used for the $\mathrm{B} \leftarrow \mathrm{X}$ transition was generated by resonance-enhanced four-wave mixing in xenon and the degree of circular polarization was determined to be $96 \%$. To analyse the deceleration and trapping experiments, the Stark effect in Rydberg states of molecular hydrogen was calculated using a matrix diagonalization procedure similar to that presented by Yamakita $e t$ al., J. Chem. Phys., 2004, 121, 1419. Particular attention was given to the prediction of zero-field positions of low- $\ell$ states and of avoided crossings between Rydberg-Stark states with different values of $\left|M_{J}\right|$. The calculated Stark maps and probabilities for diabatic traversal of the avoided crossings were used as input to Monte-Carlo particle-trajectory simulations. These simulations provide a quantitatively satisfactory description of the experimental data and demonstrate that particle loss caused by adiabatic traversals of avoided crossings between adjacent $\left|M_{J}\right|=3$ Stark states of $\mathrm{H}_{2}$ are small at principal quantum numbers beyond $n=25$. The main source of trap losses was found to be from collisional processes. Predissociation following the absorption of blackbody radiation is estimated to be the second most important trap-loss mechanism at room temperature, and trap loss by spontaneous emission is negligible under our experimental conditions.

\section{Introduction}

The desire to study molecular structure and interactions at translational temperatures below $E_{\text {kin }} / k_{\mathrm{B}}=1 \mathrm{~K}$ has recently given rise to the development of new techniques to prepare translationally cold, quantum-state-selected samples of a range of molecules ${ }^{1-3}$. Gas-phase molecular samples of this kind are important in precision spectroscopy, where resolution can be enhanced by exploiting the long interaction times that can be achieved between velocity-controlled samples and narrowbandwidth laser, millimeter-wave or microwave fields. Experimental sensitivity can also be improved by preparing molecules in selected internal quantum states. Cold samples of $\mathrm{YbF}$ and $\mathrm{PbO}$, among other molecules, are of interest in precision spectroscopic studies directed toward the detection of an electric dipole moment of the electron ${ }^{4,5}$. Precision measurements on cold $\mathrm{CO}$ and $\mathrm{HD}^{+}$samples are relevant to the determination of the proton-to-electron mass ratio and its possible variations with time ${ }^{6,7}$. In addition, high-resolution spectroscopic investigations of the structure of $\mathrm{H}_{2}$ and its isotopologues, including the ground-state ionization and dissociation energies, are of importance to fundamental studies of molecular structure and as tests of ab initio quantum chemical calculations ${ }^{8-11}$.

${ }^{a}$ ETH Zurich, Laboratory of Physical Chemistry, Wolfgang Pauli-Str. 10, Zurich, Switzerland. Fax: +41-44-632 1538; Tel: +41-44-632-4367; E-mail: frederic.merkt@xuv.phys.chem.ethz.ch
At sufficiently low translational temperatures, molecular collisions and reactions are dominated by individual angular momentum partial waves ${ }^{12}$, and quantum effects such as tunneling are expected to play an important role. For heavy species, temperatures below $1 \mathrm{mK}$ are in general necessary to enter this quantum regime. However, for light molecules such as $\mathrm{H}_{2}$, resonance effects have been predicted at temperatures on the order of $100 \mathrm{mK}$, for example in the case of the prototypical $\mathrm{F}+\mathrm{H}_{2}$ reaction ${ }^{13}$, making the preparation of translationally cold $\mathrm{H}_{2}$ very desirable in this context.

Methods to produce translationally cold, quantum-stateselected molecular samples include the association of lasercooled, ultra-cold alkali metal atoms generating dimers in their rovibrational ground state ${ }^{14-19}$ at translational temperatures on the order of $E_{\mathrm{kin}} / k_{\mathrm{B}}=100 \mu \mathrm{K}$, and approaches to preparing samples of pre-existing molecules, at translational temperatures between $10 \mathrm{mK}$ and $1 \mathrm{~K}$, either by collisional cooling with an inert buffer gas ${ }^{20}$ or by deceleration of molecular beams ${ }^{21-24}$. Molecules with a permanent electric or magnetic dipole moment in their ground state, or a low-lying metastable state, are in many cases suited to multistage Stark or Zeeman deceleration ${ }^{21-24}$. Using these methods, the decelerated samples have typical translational temperatures and densities in the ranges $10-100 \mathrm{mK}$ and $10^{6}-10^{7} \mathrm{~cm}^{-3}$, respectively.

For species with no appreciable ground state electric or magnetic dipole moment, as is the case for $\mathrm{H}_{2}$, efficient de- 
celeration and trapping can only be achieved following excitation to states with more desirable properties. Rydberg states of high principal quantum number, $n$, are in general the most suitable for this purpose because they exhibit very large electric dipole moments, $\mu \simeq n^{2} e a_{0}$, and linear Stark shifts, making control of their translational motion feasible using inhomogeneous electric fields. This approach, known as Rydberg-Stark deceleration, was first proposed by Breeden and Metcalf ${ }^{25}$ and Wing $^{26}$ with later experimental work resulting in the deflection of beams of krypton atoms ${ }^{27}$, the partial deceleration of beams of $\mathrm{H}_{2}{ }^{28,29}$ and argon ${ }^{30}$, and the reflection ${ }^{31}$ and trapping $^{32,33}$ of beams of hydrogen atoms. Because of the very large electric dipole moments involved, the adiabatic deceleration of atoms or molecules in pulsed supersonic beams from speeds on the order of $600 \mathrm{~ms}^{-1}$ to zero velocity in the laboratory frame can be achieved within distances of a few millimeters and times of a few mircoseconds, as has been demonstrated for both hydrogen atoms ${ }^{31}$ and hydrogen molecules ${ }^{34}$. The decelerated samples can be loaded into three-dimensional electrostatic traps where the effects of blackbody radiation on their fluorescence lifetimes ${ }^{35}$ and collisions with other atomic or molecular samples can be studied. Possibilities are foreseen for the magnetic trapping of paramagnetic samples after Rydberg-Stark deceleration and subsequent fluorescence or optical pumping to their ground state or a metastable state.

Two main challenges are associated with implementing Rydberg-Stark deceleration for nonhydrogenic atoms and molecules. The first arises because low-angular-momentum Rydberg states in these species are often significantly displaced in energy from the manifold of high-angular-momentum states, leading to large avoided crossings between states of adjacent $n$-manifolds in strong electric fields. At these crossings, the magnitude and orientation of the dipole associated with the states involved can change significantly, making deceleration in fields beyond these crossings infeasible. The second challenge, relevant to molecular samples, is that the lifetimes of low-angular-momentum Rydberg states are often limited by predissociation to less than $1 \mu \mathrm{s}$ and thus too short for efficient deceleration. However, by eliminating the low- $\ell$ states from the Stark manifolds, both challenges can be overcome simultaneously. The suppression of low- $\ell$ character of a Stark state can be achieved by preparing states with a value of $\left|M_{J}\right|$ sufficiently large to exclude low- $\ell$ components from the Stark states, for instance by multiphoton excitation with circularly polarized radiation.

In this article, we present in detail the photoexcitation scheme that we have exploited to prepare long-lived, nonpenetrating Rydberg states of para- $\mathrm{H}_{2}$ and to decelerate and trap hydrogen molecules at low temperature ${ }^{34}$. In particular, we describe the techniques we have developed to generate and characterize coherent, circularly polarized laser radiation in the vacuum-ultraviolet.
The success of Rydberg-Stark deceleration of molecules critically depends on the ability to carry out reliable particletrajectory simulations, which in turn cannot be achieved without a quantitative description of the Stark effect. The theoretical treatment of the Stark effect in Rydberg states of para- $\mathrm{H}_{2}$ is therefore presented in Sec. 3 with a description of how calculated Stark-maps are incorporated into a particle-trajectory simulation program that includes the Landau-Zener dynamics at avoided crossings between Stark states. The experimental data on deceleration and electrostatic trapping of para- $\mathrm{H}_{2}$ are compared with the results of particle-trajectory simulations in Sec. 4 which also includes a discussion of the mechanisms by which molecules are lost from the trap.

\section{Experimental setup and procedure}

Schematic diagrams of the multiphoton excitation sequence and the experimental setup are presented in Fig. 1. The experimental setup consists of two main parts: i) the laser system with which the radiation used in the multiphoton excitation sequence is generated and ii) a set of differentially pumped vacuum chambers including a four-wave-mixing chamber, a grating monochromator, a gas source chamber and a photoexcitation, deceleration and trapping chamber, which also contains a TOF tube and a microchannel-plate (MCP) detector (see Fig. 1(b)).

The laser system was configured to generate radiation in three different spectral regions, the vacuum ultraviolet to drive the $\mathrm{B}(v=3) \leftarrow \mathrm{X}(v=0)$ transition of $\mathrm{H}_{2}$, the visible (VIS) for the $\mathrm{I}(v=0) \leftarrow \mathrm{B}(v=3)$ transition, and the near infrared $(\mathrm{NIR})$ for the $n \ell N_{N}^{+}\left[X^{+}\left(v^{+}=0\right)\right] \leftarrow \mathrm{I}(v=0)$ transition $\left(N^{+}\right.$ and $N$ are the quantum numbers for the total angular momentum excluding spins of the ion and the Rydberg state, respectively, $v^{+}$is the vibrational quantum number of the ion and $\mathrm{X}^{+}$is the normal designation for the ground electronic state of the ion). The VUV radiation was generated by resonanceenhanced sum-frequency mixing $\left(\widetilde{v}_{\mathrm{VUV}}=2 \widetilde{v}_{\mathrm{UV}}+\widetilde{v}_{2}\right)$ in xenon using two commercial tunable dye lasers pumped by the frequency-tripled $(355 \mathrm{~nm})$ and doubled $(532 \mathrm{~nm})$ outputs of a pulsed Nd:YAG laser operated at a repetition rate of $25 \mathrm{~Hz}$. The sum-frequency generation process was enhanced at the two-photon level by fixing the doubled wave number $\left(\widetilde{v}_{\mathrm{UV}}=\right.$ $\left.2 \widetilde{v}_{1}\right)$ of the first dye laser at the position of the $5 \mathrm{p}^{5} 6 \mathrm{p}[1 / 2]_{0} \leftarrow$ $5 \mathrm{p}^{61} \mathrm{~S}_{0}$ two-photon resonance of Xe at $2 \widetilde{v}_{\mathrm{UV}}=80118.962 \mathrm{~cm}^{-1}$. The wave number of the second dye laser was then adjusted such that the VUV wave number corresponded to the $\mathrm{R}(0)$ line of the $\mathrm{B}\left(v^{\prime}=3\right) \leftarrow \mathrm{X}\left(v^{\prime \prime}=0\right)$ band of $\mathrm{H}_{2}$, i.e. to $94083.809 \mathrm{~cm}^{-1} .36$

The two laser beams ( $\widetilde{v}_{\mathrm{UV}}$ and $\left.\widetilde{v}_{2}\right)$ were overlapped using a dichroic mirror and focussed in the center of the four-wavemixing chamber at the orifice of a pulsed nozzle producing short pulses of pure xenon gas. The divergent VUV beam was then recollimated by a torroidal grating which also served the 
(a)

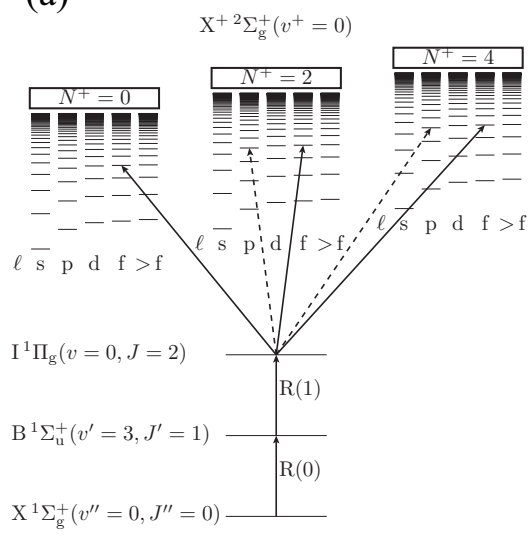

(b)

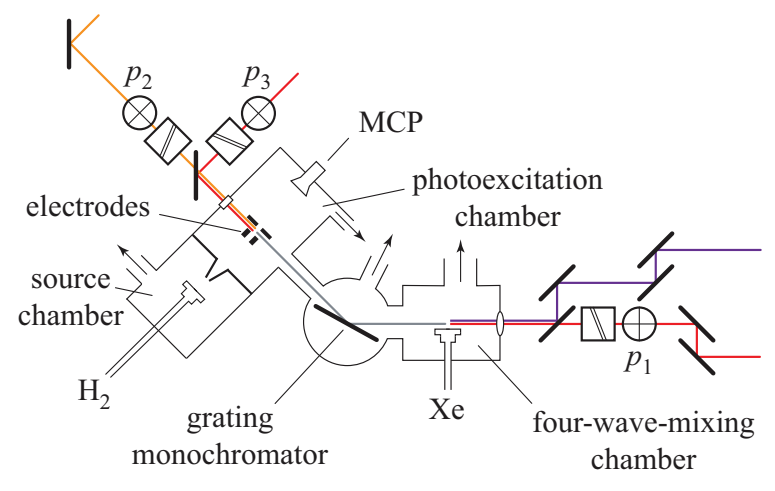

(c)

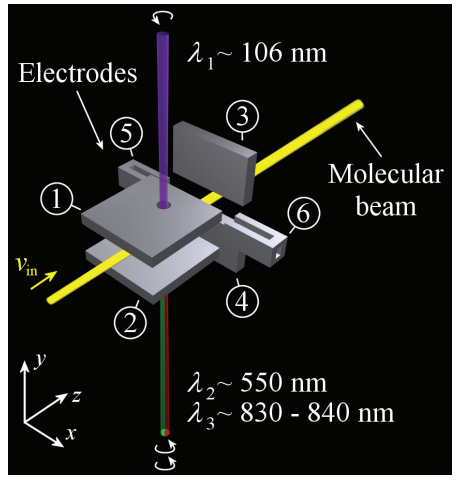

Fig. 1 (color online). (a) Resonant three-photon three-colour excitation scheme to prepare $n \mathrm{f}\left(\left|M_{J}\right|=3\right)$ Rydberg states of $\mathrm{H}_{2}$. The $\left|M_{J}\right|=3$ p-series converging to $N^{+}=2$ and 4, which are also accessible, are indicated by dashed arrows. (b) Optical layout for the generation of $\left|M_{J}\right|=3$ Rydberg states of para- $\mathrm{H}_{2}$ with three circularly polarized laser beams, prepared in the polarization stages $p_{1}, p_{2}$ and $p_{3}$, which each consist of a Glan-Taylor polarizer followed by a variable waveplate. (c) Schematic diagram of the electrode setup used to decelerate and trap translationally cold $\mathrm{H}_{2}$ Rydberg molecules.

purpose of separating the VUV beam from the fundamental beams and directing the VUV radiation toward the photoexcitation region where it intersected a pulsed, skimmed supersonic beam of $\mathrm{H}_{2}$.

Circularly polarized VUV radiation was generated by using linearly $\left(\widetilde{v}_{\mathrm{UV}}\right)$ and circularly $\left(\widetilde{v}_{2}\right)$ polarized radiation for the two lasers employed in the four-wave-mixing process. The coherent nature of the nonlinear process and the resulting necessity for the four-wave-mixing process to be an overall $\Delta M_{J}=$ 0 process ensure that the VUV radiation is circularly polarized with a helicity opposite to that of the visible laser $\left(\widetilde{v}_{2}\right)$.

The other two steps in the excitation sequence were driven by two additional commercial dye lasers with wave numbers $\widetilde{v}_{\text {VIS }}$ and $\widetilde{v}_{\text {NIR. The }} \Delta M_{J}=3$ nature of the desired three-photon excitation process necessitated that all three lasers $\left(\widetilde{v}_{\text {VUV }}, \widetilde{v}_{\text {VIS }}\right.$ and $\widetilde{v}_{\text {IIR }}$ ) propagated along the same axis (see Fig. 1(c)). For experimental convenience, the propagation direction of the VUV laser beam was chosen to be opposite to that of the other two laser beams $\left(\widetilde{v}_{\mathrm{VIS}}\right.$ and $\left.\widetilde{v}_{\mathrm{NIR}}\right)$. Circular polarization of the three lasers $\left(\widetilde{v}_{\mathrm{VIS}}, \widetilde{v}_{\mathrm{NIR}}\right.$ and $\left.\widetilde{v}_{2}\right)$ was achieved using Glan-Taylor polarizers followed by variable wave-plate compensators. Because of depolarization processes taking place at dichroic mirrors, windows and at the monochromator grating, it was necessary to precompensate the loss of circular polarization with these polarization optics. The polarization of each of the three beams was measured and optimized using a suitable set of spectroscopic transitions as explained in Sec. 4.1.

The bandwidths of the lasers were $0.15 \mathrm{~cm}^{-1}$ for the visible and NIR radiation and $\approx 0.8 \mathrm{~cm}^{-1}$ for the VUV radiation. The laser pulse energies were $\approx 0.5 \mathrm{~mJ}$ for the NIR laser,
$<1 \mathrm{~mJ}$ for the visible laser, and $\approx 2 \mathrm{~nJ}$ for the VUV laser (i.e. $\approx 10^{10}$ photon/pulse). The pulse lengths were about $2 \mathrm{~ns}$ for the VUV laser and $\approx 5-10$ ns for the NIR and visible lasers. The optical layout was chosen so that the VUV laser beam $(\approx 500 \mu \mathrm{m}$ diameter) intersected the coldest and most intense part of the supersonic beam. The sizes of the NIR and visible lasers were chosen to be larger so as to facilitate the laser alignment procedure.

In the experiments presented herein, pulsed supersonic beams of pure $\mathrm{H}_{2}$ or a mixture of $\mathrm{H}_{2}$ in $\mathrm{Kr}$ at a mixing ratio of $1: 10$ have been used. In the former (latter) case, the velocity of the beam was $2650 \mathrm{~m} / \mathrm{s}(500 \mathrm{~m} / \mathrm{s})$. The use of a pure $\mathrm{H}_{2}$ beam was particularly useful in experiments in which the $\mathrm{H}_{2}$ Rydberg molecules were detected at the microchannel plate detector, because the high velocity and shorter flight times $(\approx 90 \mu \mathrm{s})$ resulted in efficient detection within the lifetime of the excited states. For the deceleration and trapping experiments, the $\mathrm{H}_{2}: \mathrm{Kr}$ mixture was used because of its lower initial velocity.

The six-electrode deceleration and trapping setup is depicted in Fig. 1(c) and has already been described in Ref. ${ }^{33}$. Four electrodes (labeled 1-4 in Figs. 1 and 2) in a quadrupole configuration were used for deceleration and trapping in the $y$ and $z$ dimensions and confinement in the $x$ dimension was achieved with the remaining two electrodes (labeled 5 and 6) placed as end caps on each side of the trap. Time-dependent potentials were applied to electrodes 1-4 as illustrated in Fig. 2(a). Photoexcitation took place at $t=0$, at which time the field distribution is depicted in Fig. 2(b),(ii). The field at the excitation point, though weak, was spatially only weakly inhomoge- 
(a)

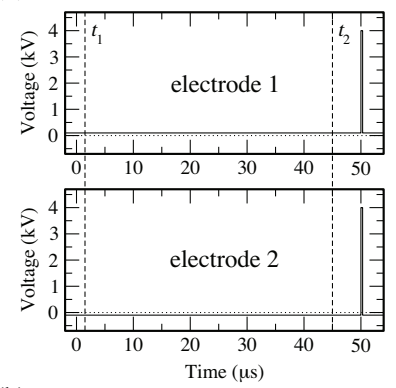

(b)

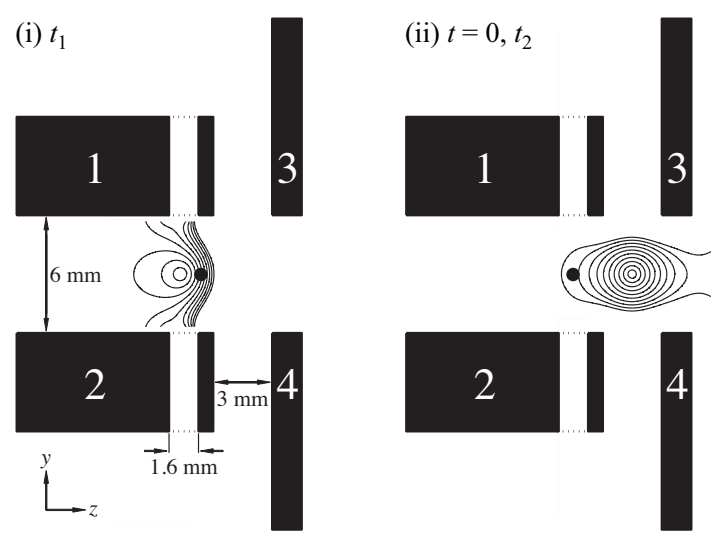

Fig. 2 (a) Time dependence of the potentials applied to electrodes 1 , 2, 3 and 4 for deceleration and trapping experiments. Electrodes 5 and 6 were kept fixed at $0 \mathrm{~V} / \mathrm{cm}$. Excitation takes place at $t=0 \mu \mathrm{s}$ and detection at $t=50 \mu \mathrm{s}$. The exponential decay of the potentials applied to electrodes 3 and 4 have a time constant $\tau_{1 / \mathrm{e}}=3.65 \mu \mathrm{s}$ and an amplitude of $2.3 \mathrm{kV}$. (b) Section of the trap in the plane defined by the laser and supersonic beams, with lines of constant electric field strength during deceleration (time $t_{1}$ ) (i) from $100 \mathrm{~V} / \mathrm{cm}$ to $1000 \mathrm{~V} / \mathrm{cm}$ in steps of $100 \mathrm{~V} / \mathrm{cm}$ and before and after deceleration (times $t=0$ and $t_{2}$ ) (ii) from $30 \mathrm{~V} / \mathrm{cm}$ to $300 \mathrm{~V} / \mathrm{cm}$ in steps of $30 \mathrm{~V} / \mathrm{cm}$ indicated in (b). The VUV, VIS and IR laser beams propagate along the $y$ direction through two holes in electrodes 1 and 2 .

neous so that well-defined Stark states could be prepared. The time dependence of the potentials ensured that (1) the Rydberg molecules were never exposed to electric fields of sufficient strength to give rise to field ionization during the deceleration process, (2) the electric field gradient along the beam propagation direction was maximized (see field distribution in Fig. 2(b),(i)), and (3) the field distribution after deceleration was identical to that at the time of photoexcitation and corresponded to a quadrupolar trap (see Fig. 2(b),(ii)). Detection was achieved by applying large positive potentials to electrodes 1 and 2 to field ionize the Rydberg states and extract the $\mathrm{H}_{2}^{+}$ions toward the microchannel plate detector. The time-offlight distribution of the $\mathrm{H}_{2}^{+}$ions enabled the reconstruction of the spatial and velocity distributions of the Rydberg molecules at the time of the field ionization, as explained in Refs. ${ }^{31,33}$.

\section{Calculations of Stark maps of $\mathbf{H}_{2}$ Rydberg states and particle-trajectory simulations}

The design, optimization and analysis of the deceleration and trapping experiments necessitate precise information on the Stark effect in Rydberg states of molecular hydrogen in the form of Stark maps, which give the energies of the optically accessible Stark states as a function of the electric field. These maps are taken as input to the particle-trajectory simulations. To calculate the Stark effect in Rydberg states of $\mathrm{H}_{2}$, the matrix diagonalization procedure used by Yamakita et al. ${ }^{29}$ has been implemented, which is itself an extension of an earlier treatment by Fielding and Softley ${ }^{37}$. The quantum defect parameters and the formulas used for the calculation of the zerofield spectra are briefly summarized in subsection 3.1. In subsection 3.2, the treatment of the Stark effect is presented with particular emphasis on the $\left|M_{J}\right|$-dependence of the Stark maps. Finally, subsection 3.3 describes how the particle-trajectory simulations were carried out.

\subsection{Quantum defects and zero-field spectra of the Ryd- berg states of $\mathbf{H}_{2}$ below the $\mathbf{X}^{+2} \Sigma_{\mathbf{g}}^{+}\left(v^{+}=0, N^{+}=0\right)$ threshold}

The matrix diagonalization procedure employed in this study is performed in a Hund's-case-(d) zero-field basis $\left|n \ell N^{+} N M_{N}\right\rangle$ where $n$ and $\ell$ are the principal and orbital angular momentum quantum numbers of the Rydberg electron, respectively, $\mathrm{N}^{+}$ is the rotational angular momentum quantum number of the $\mathrm{H}_{2}^{+}$ion core, $N$ the total angular momentum quantum number excluding spin, and $M_{N}$ the projection of $N$ onto the laser propagation axis of the laboratory-fixed reference frame (see Fig. 1(c)).

The matrix diagonalization approach used here, relies on the following assumptions: (i) The singlet-triplet interaction in para $\mathrm{H}_{2}(I=0)$ can be neglected and only singlet configurations need to be considered, so that $\vec{J}=\vec{N}=\vec{N}+\vec{\ell}$ (in the following description, $N$ and $M_{N}$ are therefore equivalent to $J$ and $M_{J}$ ). (ii) The s $\sigma-\mathrm{d} \sigma$ electronic coupling is negligible, because this coupling only becomes significant outside the region of internuclear distances relevant for $v^{+}=0$ levels of the ion $^{38}$, (iii) the $\mathrm{p} \lambda-\mathrm{f} \lambda$ interactions $(\lambda=\sigma, \pi)$ in $\mathrm{H}_{2}$ are even weaker than the s $\sigma-\mathrm{d} \sigma$ interaction, and (iv) vibrational channel interactions do not significantly perturb the spectrum in the region of interest.

A distinction is made between penetrating low- $\ell$ and nonpenetrating $\ell>3$ Rydberg states because, for $\ell \leq 3$, the Rydberg electron strongly interacts with the $\mathrm{H}_{2}^{+}$ion core. For these states, the matrix elements were calculated using the Hund'scase-(b) quantum defects $\mu_{\ell \Lambda}$ listed in Tab. 1 , where $\Lambda$ is the 
Table 1 Hund's-case-(b) quantum defects $\mu_{\ell \Lambda}$ used in the matrix diagonalization calculations ${ }^{39,40}$.

\begin{tabular}{rrrrrr}
\hline & \multicolumn{1}{c}{$\Lambda$} \\
& & 0 & 1 & \multicolumn{1}{c}{2} & \multicolumn{1}{c}{3} \\
\hline & 0 & 0.91900 & & & \\
$\ell$ & 1 & 0.18902 & -0.07925 & & \\
& 2 & 0.06700 & 0.04400 & -0.01600 & \\
& 3 & 0.01536 & 0.01247 & 0.00410 & -0.00886 \\
\hline
\end{tabular}

projection of the orbital angular momentum onto the internuclear axis $(\Lambda=\lambda$ for Rydberg states with a $\Sigma$ ion core), and transforming the quantum defect matrix from a Hund's-case(b) basis to a Hund's-case-(d) basis. For states with $\ell>3$, the Rydberg electron density in the immediate vicinity of the ion core is negligible and the quantum defects are zero to a very good approximation.

The diagonal elements of $H_{\mathrm{el}}$ in the Hund's-case-(b) basis are given by

$$
H_{\mathrm{el}}^{(\mathrm{b})}[n \ell \Lambda N, n \ell \Lambda N]=\widetilde{v}_{\mathrm{IP}}-\frac{\mathrm{Ry}}{\left(n-\mu_{\ell \Lambda}\right)^{2}}
$$

where $\widetilde{v}_{\mathrm{IP}}=124417.49113 \mathrm{~cm}^{-1}$ is the ionization energy ${ }^{41}$ of $\mathrm{H}_{2}, \mathrm{Ry}=109707.42659 \mathrm{~cm}^{-1}$ the mass-corrected Rydberg constant for $\mathrm{H}_{2}$, and $\mu_{\ell \Lambda}$ are the Hund's-case-(b) quantum defects, the numerical values of which are listed in Tab. 1.

Rotational channel interactions between series of a given $\ell(\Delta \ell=0$ and $\ell \leq 3)$ which differ in $N^{+}$by two are included as off-diagonal matrix elements

$$
H_{\mathrm{el}}^{(\mathrm{b})}\left[n \ell \Lambda N, n^{\prime} \ell \Lambda N\right]=-\frac{\mu_{\ell \Lambda}}{v_{\ell \Lambda}^{3 / 2} v_{\ell \Lambda}^{\prime 3 / 2}},
$$

where $v_{\ell \Lambda}=n-\mu_{\ell \Lambda}$ is the effective principal quantum number. The elements of the Hamiltonian matrix in Eqs. (1) and (2) are transformed from the Hund's-case-(b) basis to the Hund'scase-(d) basis using ${ }^{42,43}$

$H_{\mathrm{el}}^{(\mathrm{d})}\left[n \ell N^{+} N, n^{\prime} \ell N^{+\prime} N\right]=\sum_{\Lambda, \Lambda^{\prime}} A_{N^{+} \Lambda}^{(N \ell)} H_{\mathrm{el}}^{(\mathrm{b})}\left[n \ell \Lambda N, n^{\prime} \ell \Lambda N\right] A_{N^{+\prime} \Lambda^{\prime}}^{(N \ell)}$

where the elements $A_{N^{+} \Lambda}^{(N \ell)}$ are given by

$A_{N^{+} \Lambda}^{(N \ell)}=(-1)^{\ell+\Lambda-N^{+}}\left(\begin{array}{ccc}\ell & N & N^{+} \\ -\Lambda & \Lambda & 0\end{array}\right) \sqrt{2 N^{+}+1} \sqrt{\frac{2}{1+\delta_{\Lambda 0}}}$.

In Eq. (4), the expression in large parentheses represents a Wigner $3 j$-symbol and $\delta_{\Lambda 0}$ is a Kronecker delta. The rotational energy of the $\mathrm{H}_{2}^{+}$ion core $\left(H_{\mathrm{rot}}^{(\mathrm{d})}\left(n \ell N^{+}=2, N\right)=\right.$ $174.23160 \mathrm{~cm}^{-1}$ and $\left.H_{\text {rot }}^{(\mathrm{d})}\left(n \ell N^{+}=4, N\right)=575.43918 \mathrm{~cm}^{-1}\right)^{44}$ are included as diagonal elements of the Hamiltonian matrix in the Hund's-case-(d) basis so that the complete Hund's-case(d) matrix under field-free condition is

$$
H_{\mathrm{el}}^{(\mathrm{d})}\left[n \ell N^{+} N, n^{\prime} \ell N^{+\prime} N\right]+H_{\mathrm{rot}}^{(\mathrm{d})}\left[n \ell N^{+} N, n \ell N^{+} N\right] .
$$

To validate the procedure outlined above, the calculated zero-field energies of the $\left|n \mathrm{~s}_{0}\right\rangle,\left|n \mathrm{~d}_{2}\right\rangle,\left|n \mathrm{~s} 2_{2}\right\rangle,\left|n \mathrm{~d} 2_{0}\right\rangle$ and $\left|n \mathrm{~d} 2_{2}\right\rangle$ Rydberg levels of $\mathrm{H}_{2}$ (in the notation $\left|n \ell N_{N}^{+}\right\rangle$) were compared with the positions determined experimentally by Rottke and Welge ${ }^{45}$. Tab. 2 presents the results of this comparison in the range of $n$ values relevant to the present investigation. All calculated positions lie within less than $2.0 \mathrm{~cm}^{-1}$ of the experimental values. To obtain this level of agreement over the relevant range of states, the matrix diagonalization procedure had to include $n$ levels from 18 to 30 for $N^{+}=0$, from 14 to 20 for $N^{+}=2$ and from 10 to 14 for $N^{+}=4$. Additional tests revealed that a further increase of the range of $n$ values did not significantly change the level positions.

Table 2 Comparison between experimental ${ }^{45}$ and calculated values for the $\left|n \mathrm{~s} 0_{0}\right\rangle,\left|n \mathrm{~d} 0_{2}\right\rangle,\left|n \mathrm{~s} 2_{2}\right\rangle,\left|n \mathrm{~d} 2_{0}\right\rangle$ and $\left|n \mathrm{~d} 2_{2}\right\rangle$ Rydberg levels of $\mathrm{H}_{2}$.

\begin{tabular}{lrrr}
\hline$\left.n \ell N_{N}^{+}\right\rangle$ & $\widetilde{v}_{\text {exp. }}\left(\mathrm{cm}^{-1}\right)^{45}$ & $\widetilde{v}_{\text {calc. }}\left(\mathrm{cm}^{-1}\right)$ & $\Delta \widetilde{v}\left(\mathrm{~cm}^{-1}\right)$ \\
\hline $20 \mathrm{~s} 0_{0}$ & 124116.10 & 124116.03 & 0.07 \\
$21 \mathrm{~s} 0_{0}$ & 124144.90 & 124145.32 & -0.42 \\
$22 \mathrm{~s}_{0}$ & 124170.30 & 124170.54 & -0.24 \\
$23 \mathrm{~s}_{0}$ & 124192.10 & 124192.40 & -0.30 \\
$24 \mathrm{~s} 0_{0}$ & 124210.70 & 124211.49 & 0.79 \\
\hline $20 \mathrm{~d} 0_{2}$ & 124141.60 & 124142.52 & -0.91 \\
$21 \mathrm{~d} 0_{2}$ & 124167.60 & 124168.11 & -0.51 \\
$22 \mathrm{~d} 0_{2}$ & 124189.40 & 124190.29 & -0.89 \\
$24 \mathrm{~d} 0_{2}$ & 124225.80 & 124226.62 & -0.82 \\
$27 \mathrm{~d} 0_{2}$ & 124265.70 & 124266.72 & -1.02 \\
$29 \mathrm{~d} 0_{2}$ & 124285.10 & 124286.81 & -1.71 \\
$30 \mathrm{~d} 0_{2}$ & 124294.30 & 124295.39 & -1.09 \\
\hline $16 \mathrm{~s} 2_{2}$ & 124110.00 & 124109.03 & 0.97 \\
$17 \mathrm{~s} 2_{2}$ & 124167.60 & 124167.22 & 0.38 \\
$18 \mathrm{~s} 2_{2}$ & 124215.70 & 124215.50 & 0.20 \\
$19 \mathrm{~s} 2_{2}$ & 124255.80 & 124255.98 & -0.18 \\
\hline $16 \mathrm{~d} 2_{0}$ & 124157.60 & 124159.23 & -1.63 \\
$18 \mathrm{~d} 2_{0}$ & 124248.70 & 124250.38 & -1.68 \\
\hline $16 \mathrm{~d} 2_{2}$ & 124160.90 & 124162.20 & -1.30 \\
\hline
\end{tabular}

\subsection{Stark effect in $\mathbf{H}_{2}$}

The Hamiltonian specified in Eq. (5) describes the field-free energy levels in a Hund's-case-(d) basis in regions of the spectrum where vibrational channel interactions and s-d and p-f mixing are negligible. In the presence of an external electric field $\vec{F}=(0,0, F)$, the term $\hat{H}_{\mathrm{F}}=e F z$ must be added to the Hamiltonian. This term couples states of different orbital angular momentum quantum number according to the selection 
rule $\Delta \ell= \pm 1, \Delta N=0, \pm 1(0 \leftrightarrow 0)$ and $\Delta N^{+}=0$, resulting in off-diagonal matrix elements of the Stark Hamiltonian ${ }^{37}$

$$
\begin{aligned}
H_{\mathrm{F}}^{(\mathrm{d})}= & \left\langle v \ell N N^{+} M_{N}|e F z| v^{\prime} \ell^{\prime} N^{\prime} N^{+\prime} M_{N}^{\prime}\right\rangle \\
= & e F(-1)^{N-M_{N}+N^{\prime}+N^{+}+\ell+1} \sqrt{(2 N+1)\left(2 N^{\prime}+1\right)} \\
& \times\left(\begin{array}{ccc}
N & 1 & N^{\prime} \\
-M_{N} & 0 & M_{N}^{\prime}
\end{array}\right)\left\{\begin{array}{ccc}
\ell & N & N^{+} \\
N^{\prime} & \ell^{\prime} & 1
\end{array}\right\} \\
& \times\left\langle v \ell\|r\| v^{\prime} \ell^{\prime}\right\rangle \delta_{N^{+} N^{+\prime}}
\end{aligned}
$$

In Eq. (6), $v=n-\delta_{\ell}$ and the curly bracket denotes a Wigner $6 j$-symbol ${ }^{46}$ and $\langle\ldots\|r\| \ldots\rangle$ is a radial integral which was calculated using the Numerov method ${ }^{47}$. The two radial wavefunctions, $|v \ell\rangle$ and $\left|v^{\prime} \ell^{\prime}\right\rangle$, were integrated stepwise from $r \rightarrow$ $\infty$ inwards towards the ion core either to their inner turning points or to the polarizability radius $r_{\alpha}=3.1664 a_{0}$ of the $\mathrm{H}_{2}^{+}$ ion core ${ }^{48,49}$, whichever was encountered first. Convergence in the evalution of the radial integrals was achieved using an integration step size $\Delta r \leq 0.01 a_{0}$.

To calculate spectra, the eigenvalues $E_{k}$ and the corresponding eigenvectors $\varphi_{k}$ in an electric field are needed. A Stark state

$$
\varphi_{k}=\sum_{i} c_{k i} \phi_{i}^{(\mathrm{d})}
$$

can be described as a superposition of all Hund's-case-(d) states $\phi_{i}^{(\mathrm{d})}$ included in the calculation, where $c_{k i}$ is the coefficient of the $i$-th element of the eigenvector of state $\varphi_{k}$. Each Hund's-case-(d) state has contributions from low- $\ell$ states. The intensity of a transition from the intermediate ${ }^{1} \Pi_{g}$ level to a Rydberg-Stark state $\varphi_{k}$ is given by

$$
\left|\left\langle\varphi_{k}|\hat{\mu}| \phi_{\mathrm{I}}\right\rangle\right|^{2}=\left|\sum_{i} c_{k i}\left\langle\phi_{i}^{(\mathrm{d})}|\hat{\mu}| \phi_{\mathrm{I}}\right\rangle\right|^{2}
$$

where $\phi_{\mathrm{I}}$ is the wavefunction of the $\mathrm{I}^{1} \Pi_{\mathrm{g}}$ state. Separation of the angular momentum factors, including polarization, leads to the line strength

$$
\begin{aligned}
S= & \sum_{M}\left|\sum_{i} c_{k i}\left\langle\phi_{i}^{(\mathrm{d})}|\hat{\mu}| \phi_{\mathrm{I}}\right\rangle\right|^{2} \\
= & \sum_{M} \mid \sum_{i} c_{k i} \sum_{\Lambda} A_{N^{+}}^{(N \ell)} \sum_{q=-1}^{q=+1} \sqrt{(2 N+1)\left(2 N^{\prime}+1\right)} \\
& \times\left(\begin{array}{ccc}
N^{\prime} & 1 & N \\
-M_{N}^{\prime} & -r & M_{N}
\end{array}\right)\left(\begin{array}{ccc}
N^{\prime} & 1 & N \\
-\Lambda^{\prime} & -q & \Lambda
\end{array}\right) \\
& \left.(-1)^{M_{N}-\Lambda}\left\langle\Lambda_{i}\left|\hat{\mu}_{q}\right| \Lambda^{\prime}\right\rangle\right|^{2}
\end{aligned}
$$

where $r= \pm 1$ accounts for right and left-handed circularly polarized light and $\left\langle\Lambda_{i}\left|\hat{\mu}_{q}\right| \Lambda^{\prime}\right\rangle$ are the Hund's-case-(b) transition moments from the $\mathrm{I}^{1} \Pi_{g}$ state to the Hund's-case-(b) component of the Rydberg-Stark state $\varphi_{k}$. The Franck-Condon factor $\left|\left\langle v^{+}=0 \mid v=0\right\rangle\right|^{2}$ was assumed to be 1 because of the Rydberg character of the I state. The relative magnitude of the dipole transition moments $\left\langle\Lambda=0\left|\hat{\mu}_{q=-1}\right| \Lambda=1\right\rangle=1.0$, $\left\langle\Lambda=1\left|\hat{\mu}_{q=0}\right| \Lambda=1\right\rangle=-1.7$ and $\left\langle\Lambda=2\left|\hat{\mu}_{q=+1}\right| \Lambda=1\right\rangle=0.2$ was estimated from the relative intensities of the zero-field spectrum. However, because of the large uncertainties of these intensities, it was not possible to extract an unambiguous set of relative transition moments.

Fig. 3 shows calculated (a) $\left|M_{J}\right|=0$, (b) $\left|M_{J}\right|=1$ and (c) $\left|M_{J}\right|=3$ Stark maps of molecular hydrogen in the region around $200 \mathrm{~cm}^{-1}$ below the $\mathrm{X}^{+2} \Sigma_{g}^{+}\left(v^{+}=0, N^{+}=0\right)$ ionization threshold up to an electric field strength $F=1000 \mathrm{~V} / \mathrm{cm}$. These maps reveal spectral structures corresponding to Rydberg states converging to the $N^{+}=0,2$ and 4 ionic levels. The majority of the levels are linear combinations of high- $\ell$ states $(\ell>3)$ and are subject to the linear Stark effect characteristic of the hydrogen atom. A quadratic Stark shift is predicted for low- $\ell$ states at low fields until they join the manifolds of the high- $\ell$ states at large enough electric field strengths. Figs. 3(a-c) reveal that the low- $\ell$ states gradually disappear, and the avoided crossings between adjacent manifolds occur at progressively higher electric field strengths, with increasing $\left|M_{J}\right|$ value. Field-free energies of low- $\ell$ states determined by Rottke and Welge ${ }^{45}$ are indicated on the left-hand side of Fig. 3(a). The good agreement with our calculations confirms that $\mathrm{s} \sigma-\mathrm{d} \sigma$ mixing is negligible for Rydberg series converging to the $\mathrm{X}^{+2} \Sigma_{\mathrm{g}}^{+}\left(v^{+}=0, N^{+}=0\right)$ threshold (see also Tab. 2).

Fig. 4(a) shows the region of the $\left|M_{J}\right|=0$ Stark map around the $n=22$ and $n=23, N^{+}=0$ levels on an enlarged scale. A characteristic feature of the $\mathrm{H}_{2}$ molecule is that the $\mathrm{p}$ states lie between the $\mathrm{d}$ states and the high- $\ell$ manifold at low fields, leading to a pronounced quadratic Stark shift for the d states up to the field where these states merge with the high- $\ell$ Stark manifold. Fig. 4(b) illustrates the first few avoided crossings between the $n=22$ and $n=23$ manifolds for different $\left|M_{J}\right|$ values in the region indicated by the box in Fig. 4(a). These maps show that (i) the Stark shifts of the outermost blue-shifted state of the $n=22$ manifold and the outermost red-shifted state of the $n=23$ manifold decrease because the maximal value of $|k|$ decreases when $\left|M_{J}\right|$ increases ( $k$ represents the difference between the quantum numbers $n_{1}$ and $n_{2}$ used in the solution of the Schrödinger equation of the hydrogen atom in parabolic coordinates), and (ii) the low- $\ell$ states gradually disappear with increasing $\left|M_{J}\right|$ value, which leads to a rapid reduction of the size of the avoided crossings. The minimal energetic separation at the first avoided crossing between the $n=22$ and $n=23$ manifolds is $1.8 \mathrm{GHz}$ for $\left|M_{J}\right|=0,690 \mathrm{MHz}$ for $\left|M_{J}\right|=1,100 \mathrm{MHz}$ for $\left|M_{J}\right|=2$ and $60 \mathrm{MHz}$ for $\left|M_{J}\right|=3$. This behavior is the consequence of the gradual elimination of the core-penetrating low- $\ell$ components of the Rydberg electron wavefunction.

In time-dependent electric fields, a reduction in the size 
(a)

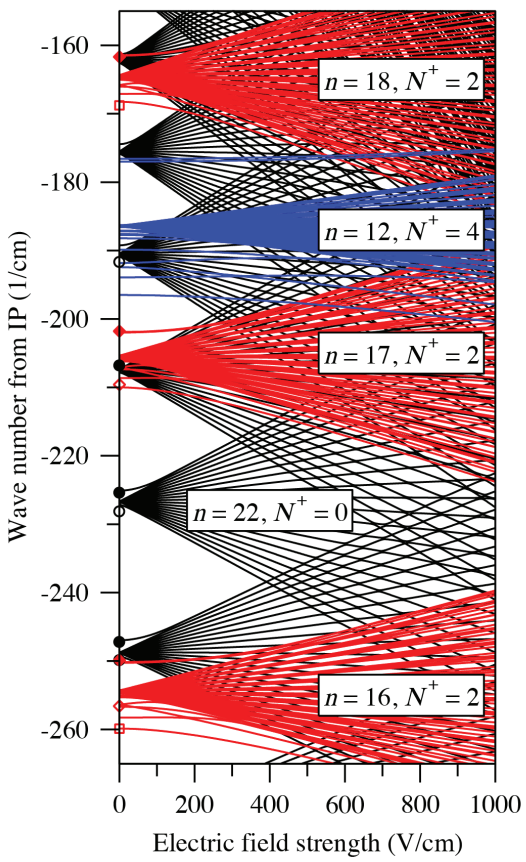

(b)

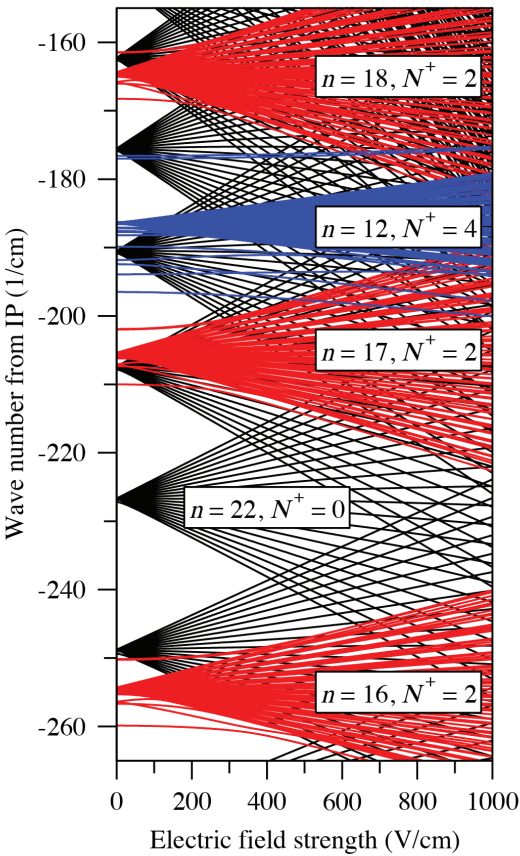

(c)

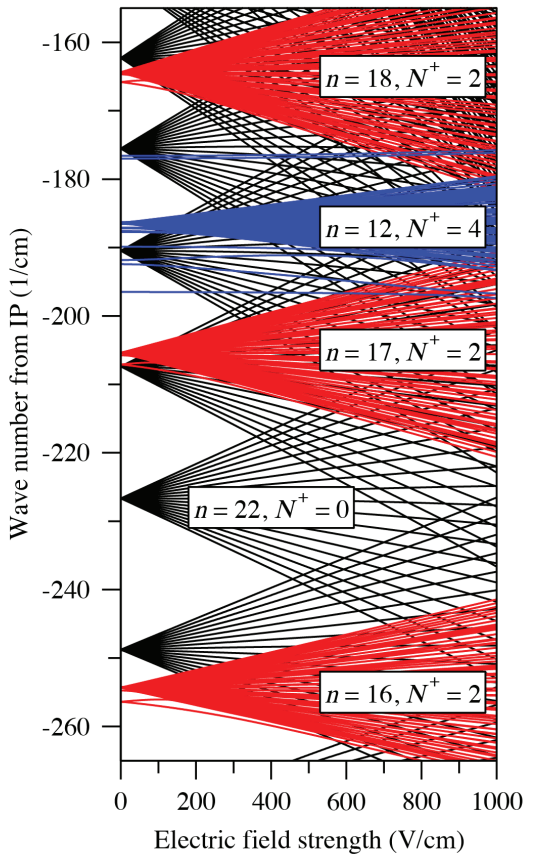

Fig. 3 (color online). (a,b,c) Calculated Stark maps for $\mathrm{H}_{2}$ including all $N^{+}=0\left(n=15-40\right.$, shown in black), $N^{+}=2(n=12-23$, shown in red) and $N^{+}=4\left(n=6-16\right.$, shown in blue) states for (a) $\left|M_{J}\right|=0$, (b) $\left|M_{J}\right|=1$ and (c) $\left|M_{J}\right|=3$. Zero-electric-field positions from Ref. ${ }^{45}$ are indicated in (a) for $\left|n \mathrm{~s} 0_{0}\right\rangle$ (full black circles), $\left|n \mathrm{~d}_{2}\right\rangle$ (open black circles), $\left|n \mathrm{~s} 2_{2}\right\rangle$ (full red diamonds), $\left|n \mathrm{~d} 2_{2}\right\rangle$ (open red diamonds) and $\left|n \mathrm{~d} 2_{0}\right\rangle$ (open red squares) states.

of the avoided crossings increases the probability of diabatic traversals ${ }^{50}$. As we shall demonstrate in Sec. 3.3, the preparation of $\left|M_{J}\right|=3$ states favours diabatic traversals and enables one to use fields larger than the field of the first crossing (this field is known as the Inglis-Teller field and scales as $\left.n^{-5}\right)^{51}$ in Rydberg-Stark deceleration experiments on $\mathrm{H}_{2}$. Increased $\left|M_{J}\right|$ values also result in prolonged lifetimes of the RydbergStark states as discussed in Sec. 4.2.

\subsection{Particle trajectory simulations}

The deceleration and trapping experiments on $\mathrm{H}_{2}$ RydbergStark states were guided and analyzed by numerical MonteCarlo (MC) particle-trajectory simulations including dynamical effects at the avoided crossings between adjacent $n$-states of the $\left|M_{J}\right|=3$ Stark manifolds of $\mathrm{H}_{2}$ (see Fig. 3(c)).
Precise information on (i) the geometry of the electrode setup, (ii) the initial position and velocity distributions of the excited Rydberg molecules, (iii) the potentials applied to the six electrodes as a function of time and (iv) the Stark map of $\mathrm{H}_{2}$ were used to calculate the trajectories in the deceleration and trapping process. The geometry of the electrode setup was identical to that described in Ref. ${ }^{33}$ with the exception of two additional $1.6 \mathrm{~mm}$-diameter holes in electrodes 1 and 2 , drilled $0.7 \mathrm{~mm}$ away from the edge in the positive $z$ direction. These holes were needed to permit the propagation of the laser beams in the direction parallel to the electric field vector and to excite $\left|M_{J}\right|=3$ Rydberg-Stark states of $\mathrm{H}_{2}$ by three-photon laser excitation. The position and velocity distributions using the methods described in Ref. ${ }^{52}$ were used in the MC particle-trajectory simulations with parameters in 
(a)

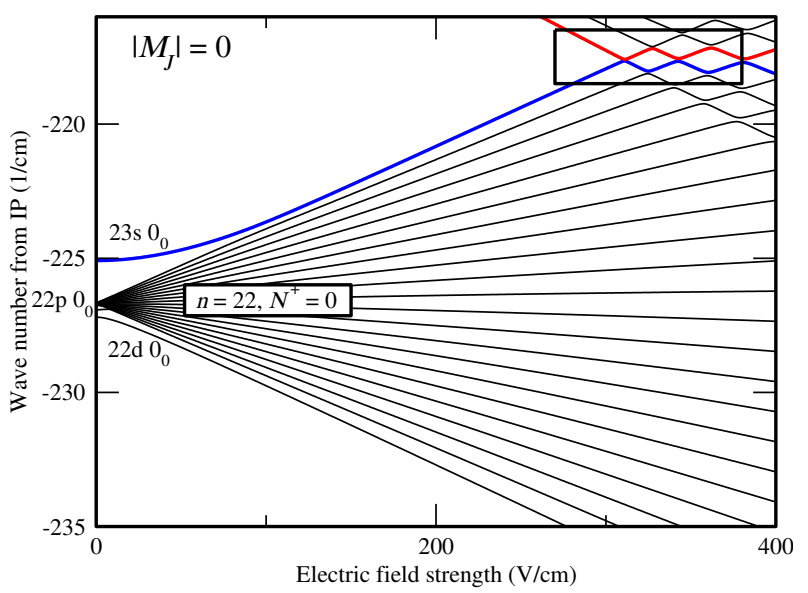

(b)
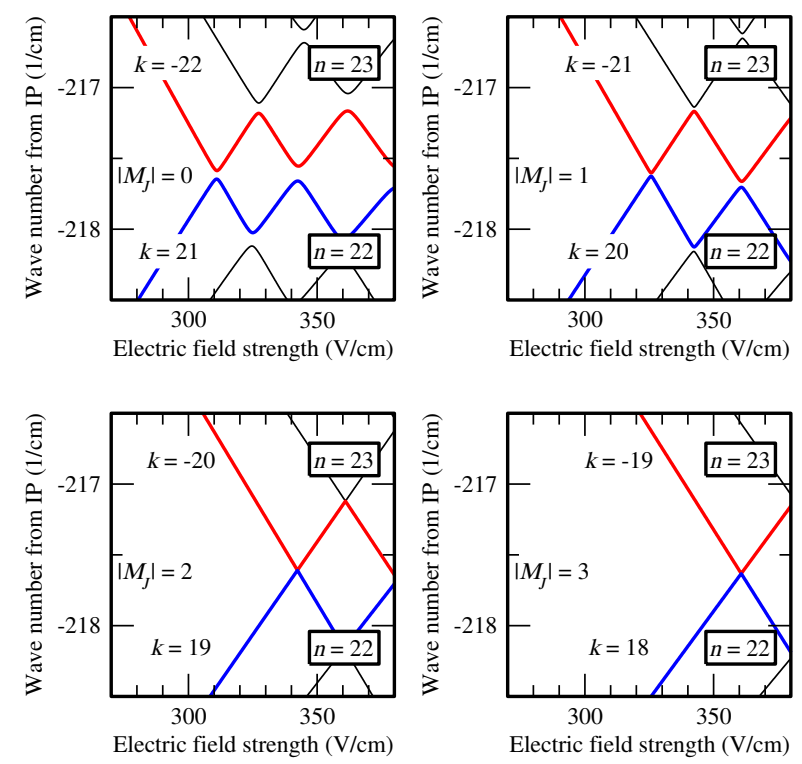

Fig. 4 (color online). (a) Calculated Stark map for $n=22, N^{+}=0$ and $\left|M_{J}\right|=0$ Rydberg states of $\mathrm{H}_{2}$. (b) Avoided crossings between the outermost $n=22$ blue-shifted and $n=23$ red-shifted Rydberg states of $\mathrm{H}_{2}$ for $\left|M_{J}\right|=0,1,2$ and 3 in the region surrounded by a frame in (a). The Stark states are labeled with the quantum number $k$ which represents the difference between the two parabolic quantum numbers $n_{1}$ and $n_{2}$.

the $x$ and $y$ dimension interchanged to account for the different propagation directions of the laser beams. The potentials applied during deceleration and trapping were measured directly in the experiment and the corresponding electric fields necessary for the calculation of the gradients and slew rates were calculated using the Simion 6 software package ${ }^{53}$. The Stark map used to evaluate the probabilities for diabatic or adiabatic traversals of the avoided crossings were calculated with the matrix diagonalization method described in Sec. 3.2 for
$N^{+}=0$ states and electric-field step sizes of $\Delta F=0.01 \mathrm{~V} / \mathrm{cm}$. Consequently, the particle-trajectory simulations did not rely on any adjustable parameters.

The dynamics at the crossings in a Stark map were assumed to be described to sufficient accuracy by two-state LandauZener theory, i.e. by

$P_{\text {diabatic }}=\exp \left\{-\frac{2 \pi\left|V_{i j}\right|^{2}}{\hbar(\mathrm{d} E / \mathrm{d} t)}\right\}=\exp \left\{-\frac{2 \pi\left|V_{i j}\right|^{2}}{\hbar(\mathrm{d} E / \mathrm{d} F)(\mathrm{d} F / \mathrm{d} t)}\right\}$

where $\mathrm{d} E / \mathrm{d} t$ is the slew rate and $V_{i j}=\Delta E / 2$ is half the size of the closest distance $\Delta E$ between the two states ( $i$ and $j$ ) involved in the avoided crossing. The slew rate $\mathrm{d} E / \mathrm{d} t$ in Eq. (10) was calculated as a product of two terms: (i) The change of energy resulting from the change of electric field strength, which can directly be determined from the calculated $\left|M_{J}\right|=3$ Stark map of $\mathrm{H}_{2}$, and (ii) the temporal change of electric field strength resulting from the motion of the molecules in the inhomogeneous field and the time-dependence of the applied voltages. Numerical convergence was reached in the simulations using time steps $\Delta t=1 \mathrm{~ns}$.

The deceleration and trapping efficiencies calculated from the MC particle-trajectory simulations were used as inputs to determine how the density of trapped molecules varied as a function of the laser excitation wave number. The relative signal at any spectral position was assumed to be proportional to the excitation efficiency to the Rydberg-Stark states. In this way, spectra were calculated that can be directly compared with spectra recorded experimentally by monitoring the field ionization of the $\mathrm{H}_{2}$ Rydberg molecules in the trap as a function of the laser wave number.

\section{Experimental results, discussion and conclu- sions}

\subsection{Generation and characterization of circularly polar- ized VUV radiation}

To quantify and optimize the degree of circular polarization of the VUV radiation, the polarization dependence of the doublyresonant three-photon ionization sequence

$$
\begin{array}{rll}
\mathrm{H}_{2}^{+} & \leftarrow \quad \mathrm{I}^{1} \Pi_{\mathrm{g}}(v=0, J=1,2) \\
& \stackrel{\mathrm{VIS}}{\leftarrow} \quad \mathrm{B}^{1} \Sigma_{u}^{+}\left(v^{\prime}=3, J^{\prime}=1\right) \\
& \stackrel{\mathrm{VUV}}{\leftarrow} & \mathrm{X}^{1} \Sigma_{g}^{+}\left(v^{\prime \prime}=0, J^{\prime \prime}=0\right)
\end{array}
$$

was exploited, in particular the fact that the two-photon transition to the $\mathrm{I}(v=0, J=1)$ level from the rovibronic ground state is forbidden when the VUV and visible lasers are both circularly polarized with the same helicity. Indeed, in this case, $\left|M_{J}\right|=2$ levels of the I state are produced, which precludes excitation of $J<2$ levels. 
Fig. 5(a) displays two measurements of the $\mathrm{I} \leftarrow \mathrm{B}$ transition recorded after excitation of the $\mathrm{B}\left(J^{\prime}=1, M_{J}^{\prime}=1\right)$ intermediate level using circularly polarized VUV radiation. The visible radiation was circularly polarized with either the same helicity as that of the VUV radiation (top panel) or the opposite helicity (bottom panel). As expected, the intensity of the transition to the $\mathrm{I}(v=0, J=1)$ state is almost negligible in the former case but strong in the latter. To quantify the degree of circular polarization, each spectrum was first calculated assuming pure circular polarization of both lasers using the following expression for the intensities of the individual transitions

$$
\begin{aligned}
I_{J \leftarrow J^{\prime}} \propto \mid & \sqrt{2 J+1} \sqrt{2 J^{\prime}+1}\left(\begin{array}{ccc}
J^{\prime}=1 & 1 & J \\
-M_{J}^{\prime}=-1 & -r & M_{J}
\end{array}\right) \\
& \times\left(\begin{array}{ccc}
J^{\prime}=1 & 1 & J \\
-\Lambda^{\prime}=0 & -q=-1 & \Lambda=1
\end{array}\right) \\
& \times\left.(-1)^{M_{J}-\Lambda}\left\langle\Lambda= \pm 1\left|\mu_{q}\right| \Lambda^{\prime}=0\right\rangle\right|^{2},
\end{aligned}
$$

with $r=1$ and $r=-1$ depending on whether the helicity of the polarization of the laser is chosen to be equal, or opposite to that of the VUV laser, respectively. The values of $q= \pm 1$ are given by the perpendicular nature of the $\Pi(\Lambda= \pm 1) \leftarrow$ $\Sigma(\Lambda=0)$ transition.

The calculated spectra are displayed in Fig. 5(b). Experimentally, the polarizations of the lasers were adjusted to minimize the intensity of the $J=1 \leftarrow J^{\prime}=1$ transition. It turned out to be impossible to completely supress the intensity of this transition (see Fig. 5(a), where the transition is marked by an asterisk). To quantify the degree of circular polarization, the measured spectrum ( $S$ in Eq. (13)) was reconstructed as a linear combination of the two spectra $S_{\circlearrowright \circlearrowright}$ and $S_{\circlearrowright \circlearrowright}$ displayed in Fig. 5(b) according to

$$
S=a \cdot S_{\circlearrowright \circlearrowright}+b \cdot S_{\circlearrowright \circlearrowright}
$$

where $a$ and $b$ are adjustable coefficients. The purity of polarization ${ }^{46}$ of the two-photon excitation

$$
C=\frac{a-b}{a+b}
$$

was established to be $96 \pm 2 \%$ by reconstructing the experimental spectra as a sum of the spectra displayed in Fig. 5(b). The reconstructed spectra are depicted in Fig. 5(c) and reproduce the experimental spectra quantitatively.

\subsection{Deceleration and trapping of $\mathbf{H}_{2}$ in $\left|M_{J}\right|=3, n=21-$ 35 Rydberg states}

The series of spectra of $\left|M_{J}\right|=3$ Rydberg states of molecular hydrogen displayed in Fig. 6 illustrate the main characteristics of the deceleration and trapping experiments. The left- (a)

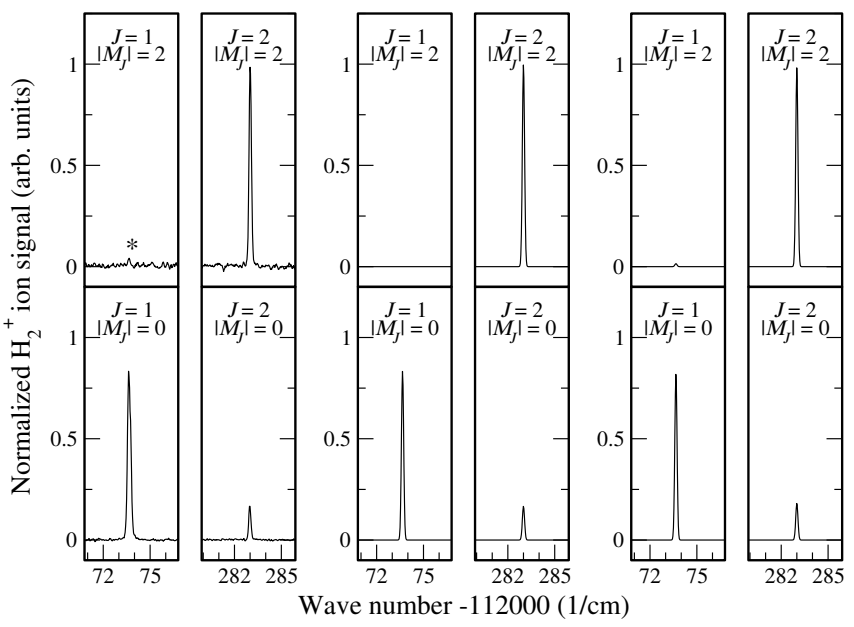

Fig. 5 (a) Experimentally observed polarization dependence of the intensity of the transitions from the $\mathrm{X}^{1} \Sigma_{\mathrm{g}}^{+}\left(v^{\prime \prime}=0, J^{\prime \prime}=0\right)$ ground state to the $\mathrm{I}^{1} \Pi_{\mathrm{g}}(v=0, J=1,2)$ states via the intermediate $\mathrm{B}^{1} \Sigma_{\mathrm{u}}^{+}\left(v^{\prime}=3, J^{\prime}=1\right)$ state. The upper (lower) trace is for the case of two circularly polarized photons having the same (opposite) helicity. The $\mathrm{I}^{1} \Pi_{\mathrm{g}}(v=0, J=1) \leftarrow \mathrm{B}^{1} \Sigma_{\mathrm{u}}^{+}\left(v^{\prime}=3, J^{\prime}=1\right) \leftarrow$ $\mathrm{X}^{1} \Sigma_{\mathrm{g}}^{+}\left(v^{\prime \prime}=0, J^{\prime \prime}=0\right)$ transition marked by an asterisk in the upper trace should be absent and its residual intensity results from an imperfection in the polarizations of the laser beams. (b) Calculations of the spectra assuming that the two lasers are perfectly circularly polarized with the same (upper trace) and opposite (lower trace) helicity. (c) Reconstruction of the experimental spectrum as a linear combination of the two spectra displayed in (b).

and right-hand sides of the figure contain experimental spectra and spectra calculated using the procedure described in Sec. 3, respectively.

The bottom trace (labelled (i)) represents a spectrum obtained by monitoring the $\mathrm{H}_{2}^{+}$ions produced by photoexcitation to $n \mathrm{f}\left|M_{J}\right|=3$ Rydberg states of $\mathrm{H}_{2}$ under field-free conditions followed by their pulsed field ionization after a delay of $3 \mu \mathrm{s}$. The spectrum consists of three series, the first and most extended one, with principal quantum number in the range $21-30$, converging to the $N^{+}=0$ ionization threshold, the second, with $n$ in the range $16-20$, converging to the $N^{+}=2$ threshold, and the third, with only the $n=12$ member in the wave number range of the figure, converging to the $N^{+}=4$ threshold. The calculated spectrum, which was generated from a stick spectrum by convolution with a gaussian line shape function of $0.8 \mathrm{~cm}^{-1}$ full width at half maximum, accurately reproduces all line positions and gives a qualitatively satisfactory description of the relative intensities.

The second trace (labelled (ii) in Fig. 6) was recorded by carrying out the excitation in the presence of an electric field of $56 \mathrm{~V} / \mathrm{cm}$ generated by applying constant potentials of $+20 \mathrm{~V}$ $(-20 \mathrm{~V}$ ) to the electrodes 1 and 4 (2 and 3) (see Fig. 2(b) for 
(a)

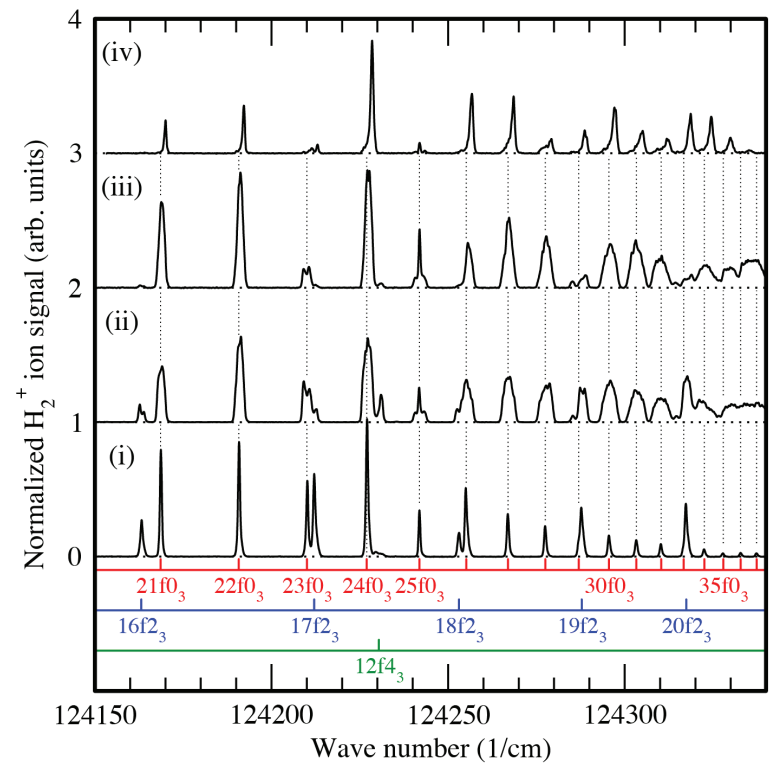

(b)

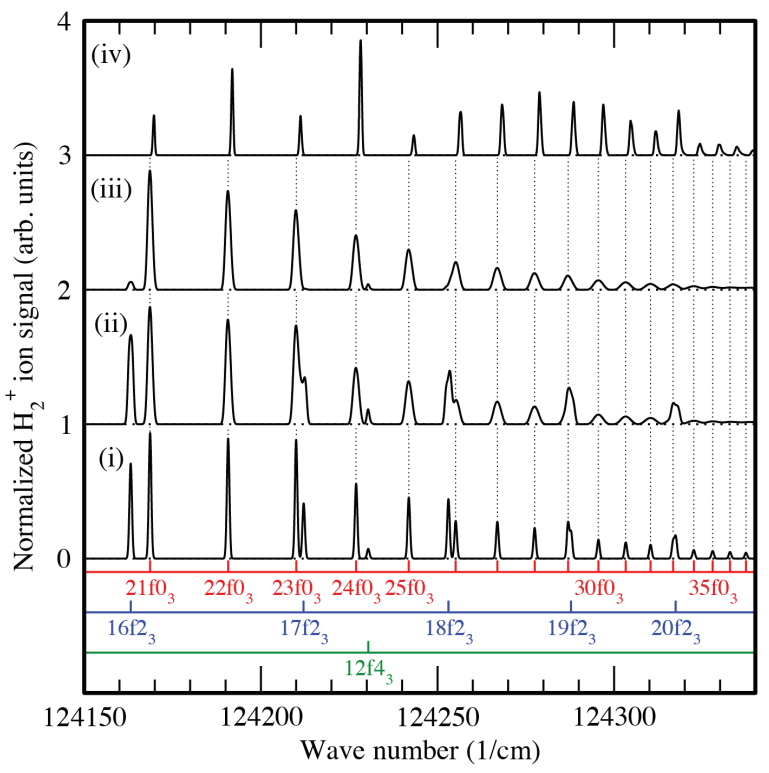

Fig. 6 (color online). (a,i) Experimentally recorded field-free spectrum of $\ell=3,\left|M_{J}\right|=3$ Rydberg states of $\mathrm{H}_{2}$. (ii) $\left|M_{J}\right|=3$ Stark spectrum recorded in an electric field of strength $56 \mathrm{~V} / \mathrm{cm}$, with pulsed-field ionization (PFI) at an ionization time $t_{\text {ion }}=3 \mu \mathrm{s}$. (iii) Stark spectrum of long-lived $\left|M_{J}\right|=3$ states recorded by measuring the signal when the neutral molecules impinge on the MCP detector after photoexcitation in a field of $56 \mathrm{~V} / \mathrm{cm}$ at $t_{\text {ion }}=90 \mu \mathrm{s}$. (iv) Low-field-seeking $\left|M_{J}\right|=3$ Rydberg-Stark states recorded by PFI following excitation in the same electric field of $56 \mathrm{~V} / \mathrm{cm}$ and deceleration and trapping for a time of $50 \mu \mathrm{s}$. The field-free states are labeled $n \ell N_{J}^{+}$, and their field-free positions are indicated by vertical dotted lines. (b,i) and (b,ii) display simulations of the recorded spectra by matrix diagonalization (see Sec. 3.2).

(iii) Simulation of the neutral $\mathrm{H}_{2}$ molecules with the same parameters used for (b,ii) but reduced lifetimes for the $N^{+}=2$ and $N^{+}=4$ states. (iv) Convolution of the simulated spectrum (b,ii) with trajectory simulations for each state $\left|n, k, N^{+}=0,\right| M_{J}|=3\rangle$ including Landau-Zener dynamics at the crossings of adjacent manifolds.

the definition of the electrode labels), under otherwise identical conditions. The electric field leads to an $n$-dependent broadening of the resonances and to an overlap of adjacent Stark manifolds beyond the $n=31, N^{+}=0$ Rydberg state. The calculations reproduce the observed Stark broadening well, but underestimate the intensities on the high-wave-number side of the spectrum.

The third spectrum (trace (iii) in Fig. 6) was also recorded following excitation in a $56 \mathrm{~V} / \mathrm{cm}$ field but using a different detection method. Instead of monitoring the pulsed-field-ionization signal, the molecules in a beam of pure $\mathrm{H}_{2}$ were allowed to fly for $\approx 90 \mu$ s and ionize at the MCP detector. The main consequence of this late detection is a reduction of the intensities of the Rydberg series converging to the $N^{+}=2$ and 4 thresholds. These states have indeed lower principal quantum numbers and are subject to a reduced Stark effect. A comparison of the relative intensities of the different series in traces (ii) and (iii) of Fig. 6(a) enabled us to make rough estimates of the lifetimes of the short-lived $N^{+}=2$ and 4 states which were both determined to be less than $30 \mu \mathrm{s}$. Values of $30 \mu \mathrm{s}$ and $20 \mu \mathrm{s}$ were used in the particle-trajectory simulations, respectively.
The top spectrum in Fig. 6 was obtained following deceleration and trapping using the voltage sequence depicted in Fig. 2(a) with a deceleration voltage of $1.7 \mathrm{kV}$, after photoexcitation in a field of $56 \mathrm{~V} / \mathrm{cm}$. To ensure that only trapped molecules contributed to the spectrum, the Rydberg states were detected by pulsed field ionization after a delay time of $50 \mu \mathrm{s}$, long enough for non-trapped molecules to have left the region of the trap. In addition, a temporal gate was placed on the $\mathrm{H}_{2}^{+}$time-of-flight trace at the time corresponding to $\mathrm{H}_{2}^{+}$ions formed at the centre of the trap.

The fact that all lines observed in this spectrum are located on the high-energy side of the zero-field resonances clearly indicates that only $\mathrm{H}_{2}$ molecules in low-field-seeking Stark states are trapped. The main reason for the observation of trapped molecules over a wide range of $n$-values between $n=$ 21 and 37 is that the magnitude of the electric dipole moment of the Stark states, which determines which states can be trapped, is equal to $\frac{3}{2} n k$ in atomic units. The lowest Rydberg states fulfilling the trapping conditions have $k \approx n$. As $n$ increases, the $k$ value of the trapped Rydberg molecules gradually decreases. The highest $n$ values at which trapping can still 
be observed is limited by the onset of field ionization during deceleration.

Stark states of identical dipole moments also have identical Stark shifts to first approximation, which explains why the shift of all lines observed in the top spectrum of Fig. 6(a) relative to the respective field-free positions $\left(\sim 1.5 \mathrm{~cm}^{-1}\right)$ does not depend on the value of $n$. These conclusions are fully supported by the results of the particle-trajectory simulations, which capture the main features of the experimental observations. The particle-trajectory simulations further indicate that, at all values of $n$ beyond 21, parts of the deceleration process take place at fields beyond the Inglis-Teller field, and that the probability of diabatic traversals of the avoided crossings in the Stark map are close to unity. This point will be discussed in more detail in subsection 4.3.

\subsection{Stark-state-resolved deceleration and trapping of $\mathrm{H}_{2}$ molecules}

The conditions under which the spectra displayed in Fig. 6 were recorded, while ideally suited to characterize the main features of the deceleration and trapping process over a broad range of $n$-values, do not allow for the resolution of individual Stark states.

To derive more information on the behaviour of individual Stark states, deceleration and trapping experiments were also carried out following excitation in a field of $278 \mathrm{~V} / \mathrm{cm}$, which is large enough to resolve the Stark states of the $n=22$ manifold of the $N^{+}=0$ series under our experimental conditions. The results of these experiments are presented in Fig. 7(a) where they are compared with the corresponding calculated spectra in Fig. 7(b).

When excitation is carried out under field-free conditions and the Rydberg states are detected by pulsed-field ionization after a delay of $3 \mu \mathrm{s}$, only one transition is observed, to the $22 \mathrm{f},\left|M_{J}\right|=3$ state (see lowest trace of Fig. 6(a)). At a field of $278 \mathrm{~V} / \mathrm{cm}$, all 19 observable Stark states with $k$ values between -18 and +18 are resolved (see trace (i) of Fig. 7(a)). Because of the inhomogeneity of the electric-field distribution and the (small) variation in the direction of the electric field with the position within the excitation volume, the calculations of relative excitation efficiencies using Eq. (9) turned out to be unreliable. Consequently, we chose to use relative excitation efficiencies determined experimentally by field ionization of the Rydberg states $3 \mu$ s after photoexcitation to normalize the particle-trajectory simulations, as depicted in Fig. 7(a,i).

Traces (ii) and (iii) in Fig. 7(a) were recorded by monitoring the pulsed-field-ionization signal of $\mathrm{H}_{2}$ Rydberg molecules in the trap $50 \mu \mathrm{s}$ after photoexcitation. When carrying out the deceleration with a deceleration voltage of $V_{\text {decel }}=1.7 \mathrm{kV}$ molecules excited to the four outermost blue-shifted Stark states $(k=12-18)$ can be trapped efficiently (see trace (ii)).
Increasing $V_{\text {decel }}$ to $2.3 \mathrm{kV}$ enables one to generate stronger gradients and thus to also efficiently trap molecules in RydbergStark states with $k=10$ (see trace (iii)), the dipole moment of which is too small for efficient trapping with $V_{\text {decel }}=1.7 \mathrm{kV}$. The experimental intensity distributions are fully accounted for by the particle-trajectory simulations (see Fig. 7(b)), from which we conclude that they capture all essential aspects of the deceleration and trapping process, including the dynamics at avoided crossings. The simulations indicate that the molecules are decelerated from $500 \mathrm{~m} / \mathrm{s}$ to zero velocity in $\approx 10 \mu \mathrm{s}$ and that the velocity distribution of the $\mathrm{H}_{2}$ molecules in the trap after $50 \mu$ s roughly corresponds to a temperature of $\sim 150 \mathrm{mK}$.

A question of central importance in view of the application of Rydberg-Stark deceleration to molecules is whether the avoided crossings between the Stark states are traversed diabatically or adiabatically during the deceleration process. The good agreement between the experimental and calculated spectra presented in Fig. 7 enable us to provide a detailed answer to this question from the calculations. The range of maximal field strengths experienced by the $\mathrm{H}_{2}$ molecules contributing to the calculated signal in Fig. 7(b) are indicated as bold lines in the Stark maps presented in Fig. 7(c). These bold lines enable one to conclude that molecules in $k=10$ and 12 Stark states traverse at most two avoided crossings during the deceleration process, once when the field increases along the trajectory and once when it decreases, and that those in $k=14-18$ Stark states traverse up to eight such crossings. When the deceleration voltage increases to $2.3 \mathrm{kV}$, the number of avoided crossings traversed by the molecules increases significantly to up to $4,10,14,14,16$ and 16 for Stark states with $k=8,10$, $12,14,16$ and 18 , respectively.

When $V_{\text {decel }}$ is initially switched on, $\mathrm{d} F / \mathrm{d} t$ is large (typically $\left.10^{8} \mathrm{~V} /(\mathrm{cm} \mathrm{s})\right)$ and more than $99.9 \%$ of the avoided crossings are traversed diabatically, except the last one which is traversed when the field approaches its maximal value. The probability of diabatic traversal of this crossing is reduced, to $70 \%$ in some cases, with large variations across the ensemble of decelerated molecules caused by the strong dependence of the slew rate during the traversal of the last crossing on the individual trajectories. During the exponential decrease of the voltage (see Fig. 2(a)), $\mathrm{d} F / \mathrm{d} t$ takes the opposite sign and most crossings are traversed in the initial phase of the exponential decay, where $|\mathrm{d} F / \mathrm{d} t|$ is large. Therefore the probability of diabatic traversal of these crossings increases again to beyond $95 \%$.

An analysis of a large number of particle trajectories at $n=22$ leads to the conclusion that in the experiment carried out with $V_{\text {decel }}=1.7 \mathrm{kV}, 97 \%$ of the molecules do not traverse any crossing during the deceleration process. Of the remaining 3\%, 33\% are finally trapped. In the experiments carried out with $V_{\text {decel }}=2.3 \mathrm{kV}$, the percentage of molecules that do not traverse any crossings reduces to $82 \%$. Of the remain- 
(a)

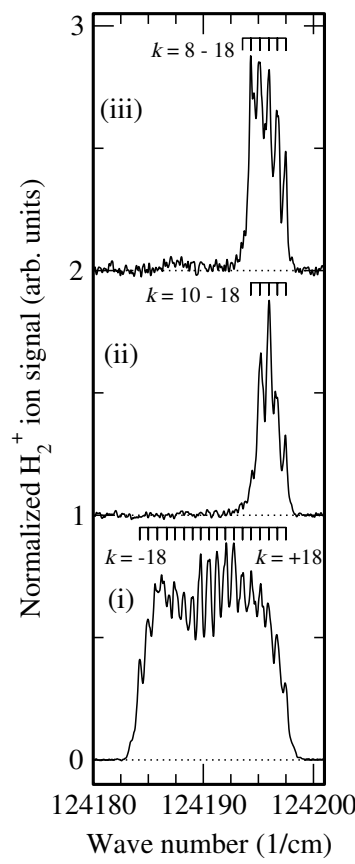

(b)

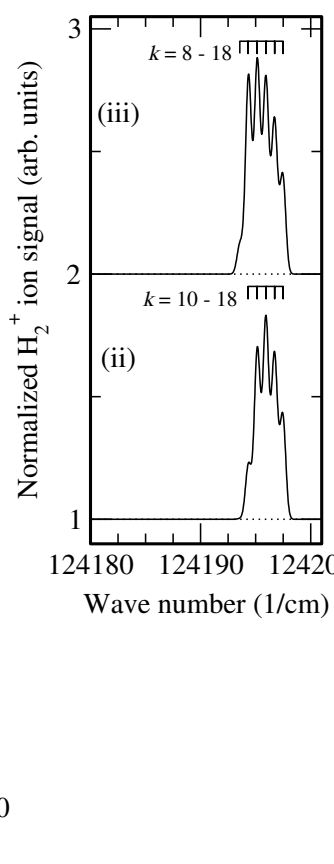

(c)
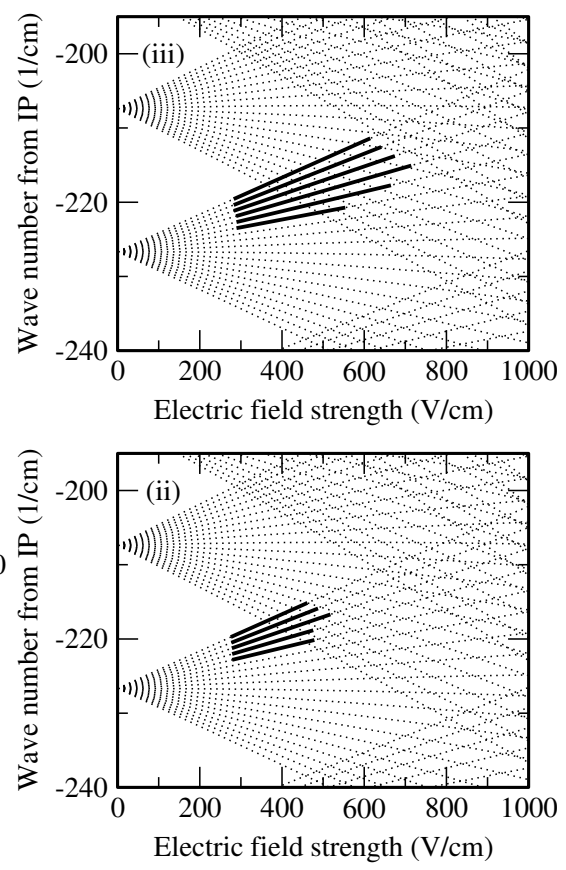

Fig. 7 (a),(i) $n=22,\left|M_{J}\right|=3$ Stark manifold recorded in an electric field of $278 \mathrm{~V} / \mathrm{cm}$ with detection at $t_{\text {ion }}=3 \mu \mathrm{s}$. (ii,iii) Spectrum of the electrostatically trapped low-field-seeking $k=10-18, n=22,\left|M_{J}\right|=3$ Stark states of $\mathrm{H}_{2}$ detected at $t_{\text {ion }}=50 \mu$ s with pulsed potentials of $1.7 \mathrm{kV}$ (ii) and $2.3 \mathrm{kV}$ (iii) applied for deceleration. (b),(ii) and (iii) Calculated spectra obtained by convolution of stick spectra with intensities proportional to the number of trapped $\mathrm{H}_{2}$ Rydberg molecules according to a particle-trajectory simulation after deceleration with potentials of $1.7 \mathrm{kV}$ (ii) and $2.3 \mathrm{kV}$ (iii), respectively. (c) Calculated $\left|M_{J}\right|=3$ Stark manifolds in the vicinity of $n=22$. The solid bars indicate the range of maximum electric field strength an ensemble of trapped $\mathrm{H}_{2}$ Rydberg molecules experience during deceleration for pulsed potentials of $1.7 \mathrm{kV}$ (ii) and $2.3 \mathrm{kV}$ (iii).

ing $18 \%, 22 \%$ are finally trapped. We thus conclude that at $n=22$ adiabatic traversals of the avoided crossings lead to at most $2 \%$ and $14 \%$ particle loss for $V_{\text {decel }}$ values of $1.7 \mathrm{kV}$ and $2.3 \mathrm{kV}$, respectively.

As $n$ increases, a much larger fraction of the molecules traverse avoided crossings during the deceleration. For instance at $n=30$, all particles traverse many crossings during the deceleration process with $V_{\text {decel }}=1.7 \mathrm{kV}$. However, the large increase in the number of traversed crossings does not lead to significant particle losses. The reason for this behaviour is that the probability of adiabatic traversals of the crossings decreases faster with $n$ than the number of crossings increases. Indeed, the magnitude of the minimal energy separation between two Stark states at an avoided crossing scales as $n^{-4}$ and the slew rate for blue-shifted Stark states scales as $n k .{ }^{54}$ When considering the magnitude of the avoided crossings, as calculated with the method described in Sec. 3, we observe that the $n^{-4}$ scaling only strictly applies for crossings between states having similar values of $|n k|$, i.e. similar dipole moments. Our calculation also indicates that the magnitude of the avoided crossing between the most blue-shifted state and the most red-shifted state of the next Stark manifold depends even more strongly on $n$.

When the probability of diabatic traversal approaches unity, the exponential function in Eq. (10) can be approximated by the first members of a Taylor series as ${ }^{55}$

$$
\begin{aligned}
P_{\text {diabatic }} & \approx 1-\frac{2 \pi\left|V_{i j}\right|^{2}}{\hbar(\mathrm{d} E / \mathrm{d} F)(\mathrm{d} F / \mathrm{d} t)} \\
P_{\text {adiabatic }} & \approx \frac{2 \pi\left|V_{i j}\right|^{2}}{\hbar(\mathrm{d} E / \mathrm{d} F)(\mathrm{d} F / \mathrm{d} t)} .
\end{aligned}
$$

Because the states that are trapped in our experiments have similar dipole moments, $n k$ and $\mathrm{d} E / \mathrm{d} F$ in Eqs. (15) and (16) are independent of $n$, so that the probability of adiabatic traversals of the avoided crossings scales as $n^{-8}$. At $n=30$, our particle-trajectory simulations indicate that the particle loss during deceleration resulting from adiabatic traversal of the avoided crossings is at most $5 \%$. 


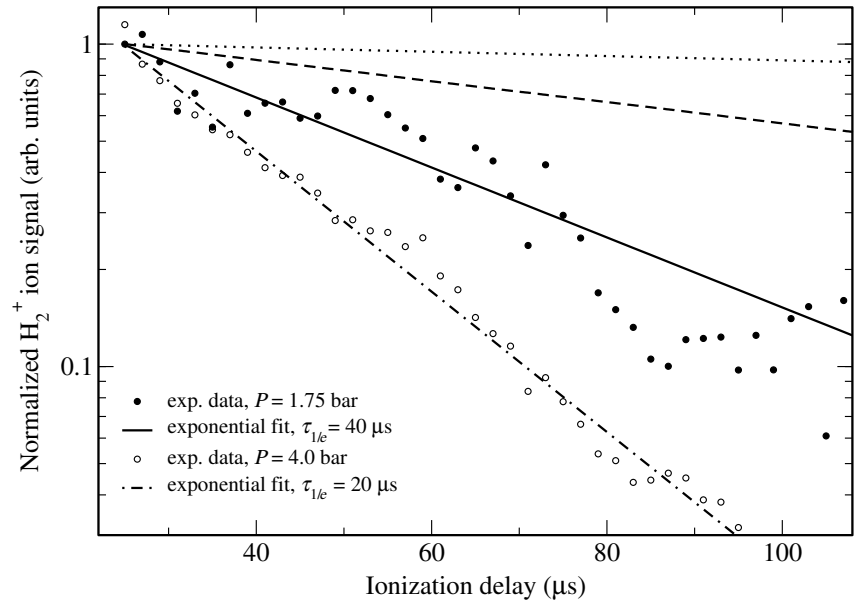

Fig. 8 Trap loss of initially prepared $n=33, k=23,\left|M_{J}\right|=3$ Rydberg-Stark states of $\mathrm{H}_{2}$ for nozzle stagnation pressures of 1.75 bar (full circles) and 4 bar (open circles). For comparison the dotted and dashed lines indicate the expected decay by fluorescence and blackbody-radiation induced predissociation, respectively.

\subsection{Trap-loss processes}

Typical measurements of trap losses carried out following excitation, deceleration and trapping of $n=33, k=23$ Stark states of $\mathrm{H}_{2}$ are presented in Fig. 8. These measurements were performed by monitoring the total field-ionization signal of the $\mathrm{H}_{2}$ molecules in the trap as a function of the time delay between photoexcitation and pulsed-field ionization. The full and open circles correspond to sets of data collected when operating the pulsed valve at stagnation pressures of $1.75 \mathrm{bar}$ and 4 bar, respectively, under otherwise identical conditions. These two data sets indicate trap decay times $\tau_{1 / e}$ of $40 \mu \mathrm{s}$ and $20 \mu \mathrm{s}$, respectively. The dotted line describes the decay one would expect if fluorescence to lower $n$ levels were the only decay process. The calculated fluorescence lifetime $\tau_{\mathrm{fl}}(n=$ $33)=640 \mu \mathrm{s}$ is significantly longer than in our previous study of atomic hydrogen $\left(\tau_{\mathrm{fl}}(n=33)=330 \mu \mathrm{s}\right)^{35}$, because fluorescence is only possible to levels with $\left|M_{J}\right|=2-4$ which must have $n \geq 3$. In the hydrogen atom experiment, the main contribution to the fluorescence rate was indeed spontaneous emission to the $n=1$ ground state because of the $v^{3}$ dependence of the Einstein $A$ coefficient for spontaneous emission. We conclude that spontaneous emission is not a significant trap-loss mechanism in the present experiments.

The second radiative process contributing to trap decay is related to blackbody-radiation-induced transitions. Although the overall depopulation rate of the initially prepared Stark state caused by blackbody-radiation-induced transitions is expected to be almost identical to that of $15400 \mathrm{~s}^{-1}$ determined in our previous study of $\mathrm{H}$ atoms, the effects of these transitions on the trap losses are qualitatively different. In $\mathrm{H}$, the dominant loss was found to be ionization by blackbody radiation, which accounts for $\approx 8 \%$ of the total depopulation rate at room temperature, leading to a trap-loss time constant of $1.4 \mathrm{~ms}$. In $\mathrm{H}_{2}$, one must also consider the possibility that blackbody-radiation-induced transitions take place to $\left|M_{J}\right|=2$ Rydberg states which can decay by predissociation. Assuming that about one third of the blackbody-radiation-induced transitions are $\Delta M_{J}=-1$ transitions leads to the conclusion that $33 \%$ of the blackbody-radiation-induced depopulation rate results in trap loss, leading to a trap decay time of $\approx 130 \mu \mathrm{s}$ displayed as dashed line in Fig. 8.

The observed trap loss rate is faster than the expected fluorescence and blackbody-radiation-induced losses just discussed. The fact that the trap-loss rate increases with increasing nozzle stagnation pressure further indicates that collisional losses are the main source of trap losses under our experimental conditions, with a possible minor contribution of slow predissociation of the $\left|M_{J}\right|=3$ levels.

Collisional losses can occur either through dipole-dipole interaction between Rydberg molecules or by interaction of the Rydberg molecules with atoms and molecules either in the background gas or in the trailing edge of the gas pulse. The $\mathrm{H}_{2}$ Rydberg molecule density of $10^{6}-10^{7} \mathrm{~cm}^{-3}$ estimated from the magnitude of the PFI signal lies on the lower end of the density range where losses from Rydberg-Rydberg collisions are expected to become significant ${ }^{56}$. In order to disentangle all decay processes, experiments are planned in a device which can be cooled to $4 \mathrm{~K}$ and can be used to trap the $\mathrm{H}_{2}$ Rydberg molecules off axis.

This work is supported by the Swiss National Science Foundation (Project No. 200020-132688) and the European Research Council advanced grant program (Project No. 228286).

\section{References}

1 M. T. Bell and T. P. Softley, Mol. Phys., 2009, 99, 107.

2 S. Y. T. van de Meerakker, H. L. Bethlem and G. Meijer, Nature Phys., 2008, 4, 595.

3 L. D. Carr, D. DeMille, R. V. Krems and J. Ye, New J. Phys., 2009, 11, 055049.

4 J. J. Hudson, B. E. Sauer, M. R. Tarbutt and E. A. Hinds, Phys. Rev. Lett., 2002, 89, 023003.

5 M. Kozlov and D. DeMille, Phys. Rev. Lett., 2002, 89, 133001.

6 H. L. Bethlem and W. Ubachs, Faraday Discuss., 2009, 142, 25.

7 S. Schiller and V. Korobov, Phys. Rev. A, 2005, 71, 032505.

8 J. Liu, H. Schmutz and F. Merkt, J. Mol. Spectrosc., 2009, 256, 111-118.

9 J. Liu, D. Sprecher, Ch. Jungen, W. Ubachs and F. Merkt, J. Chem. Phys., 2010, 132, 154301.

10 D. Sprecher, J. Liu, Ch. Jungen, W. Ubachs and F. Merkt, J. Chem. Phys., 2010, 133, 111102.

11 K. Piszczatowski, G. Lach, M. Przybytek, J. Komasa, K. Pachucki and B. Jeziorski, J. Chem. Theory Comput., 2009, 5, 3039.

12 K.-K. Ni, S. Ospelkaus, D. Wang, G. Quéméner, B. Neyenhuis, M. H. G. de Miranda, J. L. Bohn, J. Ye and D. S. Jin, Nature, 2010, 464, 1324. 
13 E. Bodo, F. A. Gianturco, N. Balakrishnan and A. Dalgarno, J. Phys. B: At. Mol. Opt. Phys., 2004, 37, 013201.

14 J. Deiglmayr, A. Grochola, M. Repp, K. Mörtlbauer, C. Glück, J. Lange, O. Dulieu, R. Wester and M. Weidemüller, Phys. Rev. Lett., 2008, 101, 133004

15 F. Lang, K. Winkler, C. Strauss, R. Grimm and J. H. Denschlag, Phys. Rev. Lett., 2008, 101, 133005.

16 K.-K. Ni, S. Ospelkaus, M. H. G. de Miranda, A. Pe'er, B. Neyenhuis, J. J. Zirbel, S. Kotochigova, P. S. Julienne, D. S. Jin and J. Ye, Science, 2008, 322, 231.

17 M. Viteau, A. Chotia, M. Allegrini, N. Bouloufa, O. Dulieu, D. Comparat and P. Pillet, Science, 2008, 321, 232.

18 S. Ospelkaus, K.-K. Ni, G. Quemener, B. Neyenhuis, D. Wang, M. H. G. de Miranda, J. L. Bohn, J. Ye and D. S. Jin, Phys. Rev. Lett., 2010, 104, 030402 .

19 J. G. Danzl, M. J. Mark, E. Haller, M. Gustavsson, R. Hart, J. Aldegunde, J. M. Hutson and H.-C. Nägerl, Nature Phys., 2010, 6, 265.

20 J. M. Doyle, B. Friedrich, J. Kim and D. Patterson, Phys. Rev. A, 1995, 52, 2515.

21 H. L. Bethlem, G. Berden and G. Meijer, Phys. Rev. Lett., 1999, 83, 1558.

22 N. Vanhaecke, U. Meier, M. Andrist, B. H. Meier and F. Merkt, Phys. Rev. A, 2007, 75, 031402(R).

23 S. D. Hogan, D. Sprecher, M. Andrist, N. Vanhaecke and F. Merkt, Phys. Rev. A, 2007, 76, 023412.

24 E. Narevicius, A. Libson, C. G. Parthey, I. Chavez, J. Narevicius, U. Even and M. G. Raizen, Phys. Rev. A, 2008, 77, 051401.

25 T. Breeden and H. Metcalf, Phys. Rev. Lett., 1981, 47, 1726-1729.

26 W. H. Wing, Phys. Rev. Lett., 1980, 45, 631-634.

27 D. Townsend, A. L. Goodgame, S. R. Procter, S. R. Mackenzie and T. P. Softley, J. Phys. B: At. Mol. Opt. Phys., 2001, 34, 439-450.

28 S. R. Procter, Y. Yamakita, F. Merkt and T. P. Softley, Chem. Phys. Lett., 2003, 374, 667-675.

29 Y. Yamakita, S. R. Procter, A. L. Goodgame, T. P. Softley and F. Merkt, J. Chem. Phys., 2004, 121, 1419-1431.

30 E. Vliegen, H. J. Wörner, T. P. Softley and F. Merkt, Phys. Rev. Lett., 2004, 92, 033005.

31 E. Vliegen and F. Merkt, Phys. Rev. Lett., 2006, 97, 033002.

32 E. Vliegen, S. D. Hogan, H. Schmutz and F. Merkt, Phys. Rev. A, 2007, 76, 023405 .
33 S. D. Hogan and F. Merkt, Phys. Rev. Lett., 2008, 100, 043001.

34 S. D. Hogan, Ch. Seiler and F. Merkt, Phys. Rev. Lett., 2009, 103, 123001.

35 Ch. Seiler, S. D. Hogan, H. Schmutz, J. A. Agner and F. Merkt, Phys. Rev. Lett., 2011, 106, 073003.

36 J. Philip, J. P. Sprengers, T. Pielage, C. A. de Lange, W. Ubachs and E. Reinhold, Can. J. Chem., 2004, 82, 713-722.

37 H. H. Fielding and T. P. Softley, Chem. Phys. Lett., 1991, 185, 199-205.

38 S. C. Ross and Ch. Jungen, Phys. Rev. A, 1994, 49, 4353-4363.

39 A. Osterwalder, A. Wüest, F. Merkt and Ch. Jungen, J. Chem. Phys., 2004, 121, 11810-11838.

40 A. Osterwalder, PhD thesis, Eidgenössische Technische Hochschule Zürich, ETH Zürich, CH-8093 Zürich, Switzerland, 2002.

41 J. Liu, E. J. Salumbides, U. Hollenstein, J. C. J. Koelemeij, K. S. E. Eikema, W. Ubachs and F. Merkt, J. Chem. Phys., 2009, 130, 174306.

42 G. Herzberg and Ch. Jungen, J. Mol. Spectrosc., 1972, 41, 425-486.

43 Ch. Jungen and G. Raseev, Phys. Rev. A, 1998, 57, 2407-2419.

44 R. E. Moss and L. Valenzano, Mol. Phys., 2003, 101, 2635.

45 H. Rottke and K. H. Welge, J. Chem. Phys., 1992, 97, 908-926.

46 R. N. Zare, Angular Momentum, John Wiley \& Sons, New York, 1988.

47 M. L. Zimmerman, M. G. Littman, M. M. Kash and D. Kleppner, Phys. Rev. A, 1979, 20, 2251-2275.

48 P. L. Jacobson, D. S. Fisher, C. W. Fehrenbach, W. G. Sturrus and S. R. Lundeen, Phys. Rev. A, 1997, 56, 4361.

49 P. L. Jacobson, D. S. Fisher, C. W. Fehrenbach, W. G. Sturrus and S. R. Lundeen, Phys. Rev. A, 1998, 57, 4065.

50 J. R. Rubbmark, M. M. Kash, M. G. Littman and D. Kleppner, Phys. Rev. A, 1981, 23, 3107-3117.

51 T. F. Gallagher, Rydberg Atoms, Cambridge University Press, Cambridge, 1994.

52 E. Vliegen, P. Limacher and F. Merkt, Eur. Phys. J. D, 2006, 40, 73-80.

53 D. A. Dahl, Simion 3D Version 6.0, computer program, Locked Martin Idaho Technologies, Idaho National Engineering and Environmental Laboratory, 1995.

54 I. V. Komarov, T. P. Grozdanov and R. K. Janev, J. Phys. B: At. Mol. Phys., 1980, 13, L573-L576.

55 W. A. Chupka, J. Chem. Phys., 1993, 98, 4520-4530.

56 N. Saquet, A. Cournol, J. Beugnon, J. Robert, P. Pillet and N. Vanhaecke, Phys. Rev. Lett., 2010, 104, 133003. 